\title{
Initial experience in self-monitoring of intraocular pressure
}

European Journal of Ophthalmology 0 (0) 1-7

(C) The Author(s) 2020

(c) (i) (8)

Article reuse guidelines:

sagepub.com/journals-permissions DOI: 10.1 |77/I | 20672|20920217

journals.sagepub.com/home/ejo

(S)AGE

\author{
Emily McGarva', Jane Farr', Priya Dabasia ${ }^{2}$, \\ John G Lawrenson ${ }^{2,3}$ and Ian E Murdoch ${ }^{1,3}$ (D)
}

\begin{abstract}
Background/aims: Diurnal variation in intraocular pressure (IOP) is a routine assessment in glaucoma management. Providing patients the opportunity to perform self-tonometry might empower them and free hospital resource. We previously demonstrated that $74 \%$ of patients can use the Icare ${ }^{\circledR}$ HOME tonometer. This study further explores Icare ${ }^{\circledR}$ HOME patient self-monitoring.

Methods: Patients were trained by standard protocol to use the Icare ${ }^{\circledR}$ HOME rebound tonometer. Patient selftonometry was compared to Goldmann applanation tonometry (GAT) over one clinical day. Following this, each patient was instructed to undertake further data collection that evening and over the subsequent two days.

Results: Eighteen patients (35 eyes) participated. Good agreement was demonstrated between GAT and Icare ${ }^{\circledR}$ HOME for IOPs up to $15 \mathrm{~mm} \mathrm{Hg}$. Above this IOP the Icare ${ }^{\circledR}$ tended to over-read, largely explained by 2 patients with corneal thickness $>600 \mathrm{um}$. The mean peak IOP during 'clinic hours' phasing was $16.7 \mathrm{~mm} \mathrm{Hg}$ and $18.5 \mathrm{~mm} \mathrm{Hg}(p=0.24)$ over three days. An average range of $5.0,7.0$ and $9.8 \mathrm{~mm} \mathrm{Hg}$ was shown during single day clinic, single day home and three day home phasing respectively $(\mathrm{p}=<0.00 \mathrm{I})$. The range of IOP was lower in eyes with prior trabeculectomy $(6 . \mathrm{l} \mathrm{mm} \mathrm{Hg}$ vs $12.2 \mathrm{~mm} \mathrm{Hg}$ ). All patients undertook one reading in the early morning at home with an average of 4.8 readings during, and 3.1 readings after office hours.

Conclusions: This small study shows that self-tonometry is feasible. The findings from home phasing demonstrated higher peak and trough IOPs, providing additional clinical information. Home phasing is a viable alternative. The costeffectiveness of this approach has yet to be addressed.
\end{abstract}

\section{Keywords}

Glaucoma, self-tonometry, phasing, methods comparison study

Date received: 2 April 2019; accepted: 27 March 2020

\section{Introduction}

As intraocular pressure (IOP) is the primary modifiable risk factor in glaucoma treatment, ${ }^{1}$ IOP readings are a critical component of glaucoma management. While establishing diagnosis and treatment, a patient may be asked to attend for a full day of IOP measurements (phasing) as fluctuating and raised IOP are more marked in those with glaucoma ${ }^{2}$ and are associated with primary open angle glaucoma progression. ${ }^{3-6}$

Day phasing is generally performed with the accepted gold standard Goldmann applanation tonometry $(\mathrm{GAT})^{7}$ by a trained healthcare professional (e.g. nurse, optometrist or doctor) using topical anaesthesia and a device mounted on a slit lamp. For logistical reasons, phasing is generally limited to standard clinic working hours. This means peaks and troughs of IOP, known to also occur in normal individuals, 8,9 may be missed if they occur outside working hours.

An alternative to clinic day phasing is home monitoring. This could allow a wider range of phasing times,

\footnotetext{
'Moorfields Eye Hospital NHS Foundation Trust, London, UK

${ }^{2}$ Division of Optometry and Visual Sciences, City, University of London, London, UK

${ }^{3}$ UCL Institute of Ophthalmology, London, UK
}

\section{Corresponding author:}

lan E Murdoch, UCL Institute of Ophthalmology, I I-43 Bath Street, London ECIV 9EL, UK.

Email: i.murdoch@ucl.ac.uk 
less interference in the patient's daily activities and reduced strain on busy clinics. The first report of home tonometry in $1965^{10}$ involved Schiötz tonometry. This requires the use of topical anaesthesia, as well as an additional person to perform the measurement, and has the potential risk of corneal abrasion.

The concept of self-tonometry was conceived in 1967. ${ }^{11}$ Despite many subsequent developments, selfphasing at home has not yet reached the stage of replacing IOP phasing in the clinical setting. Home monitoring is now commonplace in relation to the diagnosis and management of systemic hypertension, ${ }^{12}$ diabetes $^{13}$ and many other conditions. The concept of IOP home monitoring is now more feasible due to the design of tonometers that do not require topical anaesthesia.

The Icare $^{\circledR}$ HOME tonometer is designed specifically for self-tonometry. This new and updated model of the Icare ${ }^{\circledR}$ ONE tonometer uses the same rebound technology and principles whereby IOP is calculated from the deceleration of the probe and corneal contact time. $^{14}$

The Icare ${ }^{\circledR}$ HOME includes EyeSmart eye recognition technology to assign each measurement to the right or left eye and EasyPos alignment features to make the device more user friendly.

There have been several studies investigating the accuracy and usability of Icare $^{\circledR}$ devices. However, to date there are few reports of the Icare ${ }^{\circledR}$ HOME being used as intended in a 'home' setting, away from the typical clinic environment. This study aimed to evaluate the feasibility of performing self-tonometry for phasing in a home setting and the clinical usefulness of performing self-tonometry in this setting by establishing fluctuations in IOP and the daily peak of IOP. It also provides further information in relation to the Icare ${ }^{\circledR}$ HOME's self-tonometry readings compared to in-clinic GAT phasing.

\section{Materials and methods}

Patients were recruited using the following inclusion criteria: aged 18 years or older with a diagnosis of ocular hypertension (OHT) (healthy disc, full visual fields (Humphrey HFA 24-2 SITA) and IOP $>21 \mathrm{~mm} \mathrm{Hg}$ on at least two separate occasions), or open angle glaucoma (OAG), or angle closure glaucoma (ACG) (pathological cupping of optic disc with matching visual field loss in the presence of an open or occludable angle respectively), willing consent to inclusion in the study, and ability to use the Icare ${ }^{\circledR}$ HOME tonometer (Icare Finland Oy. Vantaa, Finland).

Exclusion criteria consisted of one or more of the following: inability to speak fluent English, inability to reliably use Icare $^{\circledR}$ HOME tonometer, and ocular abnormalities likely to give rise to artefactual IOP measurements (for example severe corneal scarring). This study was approved by the School of Health Sciences Research Ethics Committee, City University of London, and complied with the tenets of the Declaration of Helsinki. Written and informed consent was obtained from all participants prior to taking part in the study.

Patients followed two study phases. Phase one involved a standardised training protocol that allowed the patients to build familiarity and competency with the Icare ${ }^{\circledR}$ HOME tonometer. This was followed by baseline data collection including demographic information and a brief history including previous ocular history. LogMAR visual acuity was measured with the subject's current distance spectacles. Central corneal thickness (CCT) was recorded using a Pachmate 2 pachymeter (DGH Technology, Inc. Exton, Pennsylvania, USA).

The training protocol was developed in line with manufacturer recommendations and is described in detail in a previous report. ${ }^{15}$ It is summarised in Figure 1.

The patient obtained three consecutive IOP measurements with the Icare $^{\circledR}$ HOME tonometer followed immediately by one reading taken by an optometrist and three consecutive GAT measurements, while masked to the patient's self-Icare ${ }^{\circledR}$ HOME results. The Goldmann dial was set to $10 \mathrm{~mm} \mathrm{Hg}$ before each reading was taken and the median of each set of readings was used for analysis. ${ }^{16}$ Patients were classified as competent in self-tonometry according to the following criteria:

1. The Icare ${ }^{\circledR}$ HOME reading taken by the optometrist and the first of the 3 readings taken by the patient differ by $5 \mathrm{~mm} \mathrm{Hg}$ or less.

2. The range ( $\max$ to $\min$.) of the first 2 readings taken by the patient is:

a. $5 \mathrm{~mm} \mathrm{Hg}$ or less if the first reading is $7-23 \mathrm{~mm} \mathrm{Hg}$ OR

b. $7 \mathrm{~mm} \mathrm{Hg}$ or less if the first reading is $>23 \mathrm{~mm} \mathrm{Hg}$

3. The positioning of the Icare ${ }^{\circledR}$ HOME tonometer was correct during self-use as determined by the optometrist.

Phase two involved three days of data collection. On the first day (approximate clinic hours 09:00-16:30) hourly IOP assessments were made in-clinic. The subject took three consecutive Icare $^{\circledR}$ HOME measurements followed by the optometrist taking three consecutive GAT readings. The Goldmann tonometer's calibration was verified each day. The subject was then provided with an Icare ${ }^{\circledR}$ HOME tonometer, probes, written information, spare batteries and a feedback sheet. The subject was instructed to perform three consecutive self-tonometry readings at two hourly 


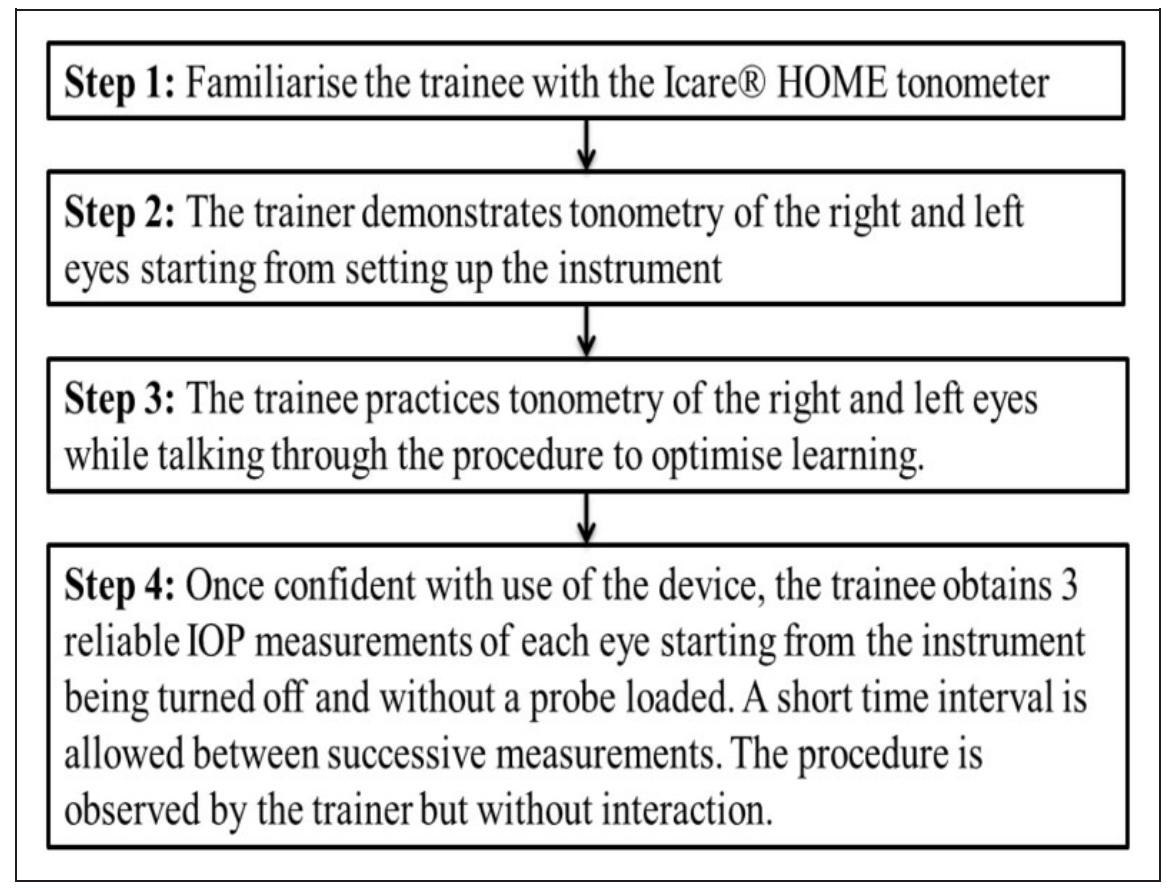

Figure I. Standardised training procedure for the use of the Icare ${ }^{\circledR}$ HOME tonometer.

intervals on the same evening as the clinic phasing and also over a subsequent two day period. It was requested that patients aimed to include early morning and late evening measurements. The data collection, outside of the clinic, is referred to as 'home phasing' and encompasses the patient performing self-tonometry in a home environment, as well as any other mobile location that they felt was appropriate to them. For example, this could be while they were at work or socialising.

Home phasing IOP data (including time, date and eye measured) was stored within the tonometer and not visible to the patient. On return of the tonometer, the data was downloaded to a computer with Icare ${ }^{\circledR}$ Link software for storage and viewing.

\section{Analysis}

For all analyses the median IOP reading was taken. Bland and Altman ${ }^{17}$ plots were used for the observer comparison stage of this study. For the comparison of the continuous variable of IOP, descriptive statistics were used (average, range, peak and trough) together with paired t-tests with a $\mathrm{p}$ value of $\leq 0.05$ being taken as significant (data distribution was checked for normality prior to this test).

\section{Results}

Eighteen patients (35 eyes) completed the observer comparison phasing day in clinic office hours $(100 \%$ training protocol pass rate). Seventeen patients
Table I. Clinical data from the 18 patients (35 eyes) who completed phase one and phase two.

\begin{tabular}{ll}
\hline Measurement & Mean (SD) \\
\hline Visual Acuity (logMAR) & $0.05(0.15)$ \\
Age (years) & $69(6.6)$ \\
Gender & $39 \%$ female \\
Diagnosis & I4 Open Angle Glaucoma \\
& I Angle Closure Glaucoma \\
& 3 Ocular Hypertension \\
Prior Trabeculectomy & 7 eyes $(6$ patients) \\
Mean Refractive Error (DS) & $-0.08(2.38)$ \\
Vertical Palpebral Aperture (mm) & $10.1(1.5)$ \\
CCT ( $\mu$ m) & $543(34)$ \\
GAT IOP (mmHg) & $13.4(4.8)$ \\
iCare IOP (mmHg) & $14.3(6.1)$ \\
\hline
\end{tabular}

CCT: Central corneal thickness; GAT: Goldmann applanation tonometry; IOP: intraocular pressure. DS: Dioptres.

(34 eyes) carried out home phasing for the subsequent two days and one patient ( 2 eyes) for one day. The demographic and clinical findings for all participants are shown in Table 1.

A Bland Altman plot comparing GAT and Icare ${ }^{\circledR}$ Home shows good agreement up to $15 \mathrm{~mm} \mathrm{Hg}$ then suggests an over-read for the Icare $^{\circledR}$ HOME for higher IOPs (Figure 2). The over-read increases with increasing IOP $\left(\mathrm{r}^{2}=0.29 \mathrm{p}<0.001\right)$. This finding is in part, but not wholly, explained by two patients (one of whom had prior trabeculectomy) with an average CCT 


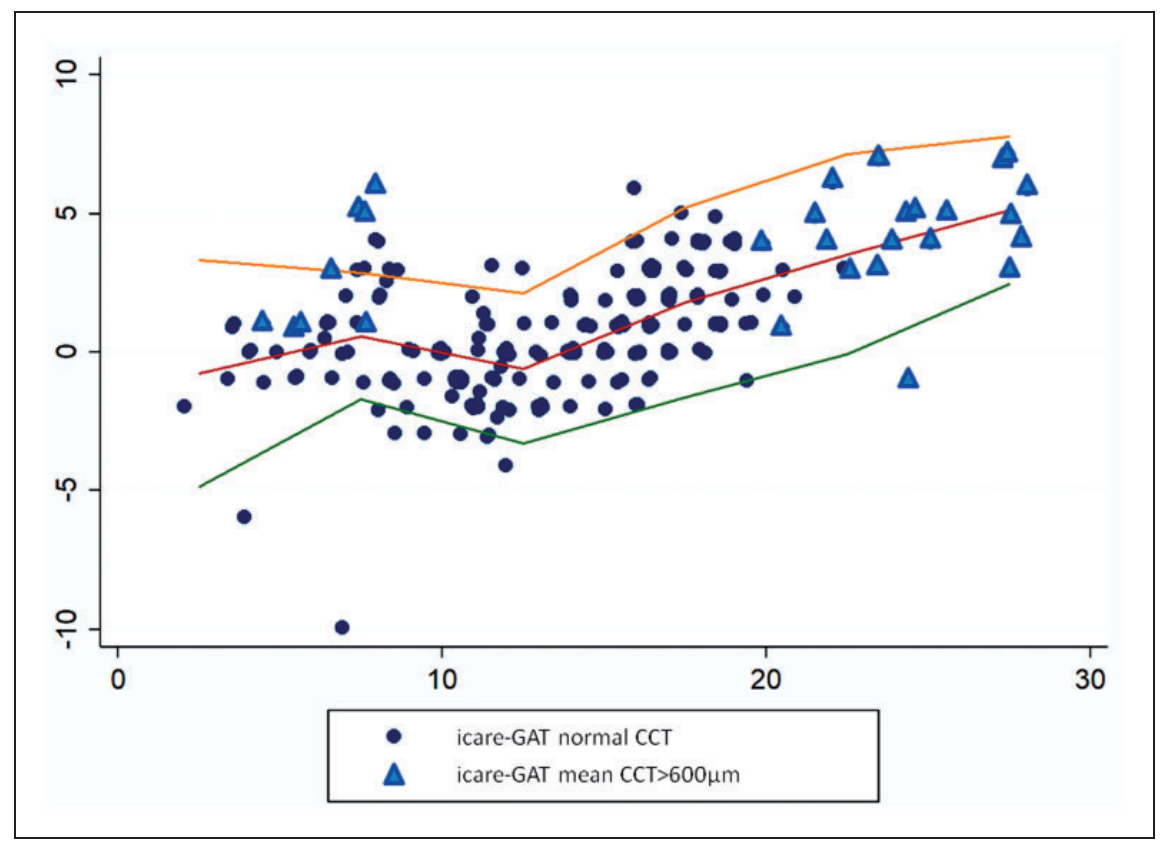

Figure 2. Bland Altman agreement between GAT and Icare ${ }^{\circledR}$ HOME. The horizontal axis is the mean reading and the vertical axis is the self Icare ${ }^{\circledR}-$ Goldmann reading. The horizontal line shows the average result by $5 \mathrm{mmHg}$ group. A jitter plot is used to highlight multiple corresponding data points. The triangular points represent those taken from eyes with CCT $>600 \mu \mathrm{m}$.

of $>600 \mathrm{um}$, which exceeds the recommended range for Icare $^{\circledR}$.

The mean peak IOP recorded with the Icare $^{\circledR}$ HOME during clinic self-tonometry phasing was $16.7 \mathrm{~mm} \mathrm{Hg}$. The mean peak with all day recording was marginally higher $(17.1 \mathrm{~mm} \mathrm{Hg}, \mathrm{p}=0.02)$. The mean peak using data from three days recording was higher again $(18.5 \mathrm{~mm} \mathrm{Hg} \mathrm{p}<0.001)$.

The range of IOP recorded was then examined. Single day clinic self-tonometry phasing with the Icare $^{\circledR}$ HOME showed an average range of $5.0 \mathrm{~mm} \mathrm{Hg}$. All day recording showed a larger range of $7.0 \mathrm{~mm} \mathrm{Hg} \quad(\mathrm{p}<0.001)$ and three day readings showed a larger range again of $9.8 \mathrm{~mm} \mathrm{Hg}(\mathrm{p}<0.001)$.

Table 2 shows the variation by diagnosis and surgical intervention. Of note is that those with OHT had the highest range in IOP $(12.2 \mathrm{~mm} \mathrm{Hg}, \mathrm{p}=0.001)$ whereas the eyes with prior trabeculectomy surgery had the lowest $(6.1 \mathrm{~mm} \mathrm{Hg}, \mathrm{p}=0.29)$.

The timings of IOP measurements in the clinic and home settings were compared. 6-7 readings were taken between 09:00 and 16:30 in the clinical setting. When performing home phasing all patients undertook one reading in the morning, generally between 07:00 and 08:00, with an average of 4.8 readings during standard office hours (i.e. 09:00-17:00) and 3.1 readings taken after 17:00. The average peak IOP was highest during standard office hours $(16.4 \mathrm{~mm} \mathrm{Hg})$, being $14.7 \mathrm{~mm} \mathrm{Hg}$ in early morning and $15.1 \mathrm{~mm} \mathrm{Hg}$ in the evening.
During home phasing a total of 306 self-tonometry readings were taken. $38(12.4 \%)$ were prior to clinic office hours, $153(50 \%)$ during office hours and 115 $(37.6 \%)$ after office hours.

\section{Discussion}

All patients entering the study successfully completed the self-tonometry training protocol (phase one) in at least one eye and subsequently all were classed as competent in self-tonometry and were able to move onto phase two with data collection. Our previous study found that $74 \%$ of subjects were able to correctly perform self-tonometry with the Icare ${ }^{\circledR}$ HOME. ${ }^{15}$ This also aligns with a similar study that found a $73 \%$ rate of success in training. ${ }^{18}$ These studies did not investigate 'home' setting self-tonometry.

It is likely that this study has some bias inherent in the phase one training pass rates of $100 \%$. This bias may stem from a high level of patient motivation from the outset due to their intended commitment to the three days of home phasing. In addition, all patients had steady hands, a sufficiently large palpebral aperture and a reasonable level of vision that allowed them to competently perform self-tonometry in at least one eye.

Although recent studies have reported that the Icare $^{\circledR}$ HOME tonometer can be correctly used by the majority of patients, ${ }^{15,18-20}$ there have been 
Table 2. IOP variation by diagnosis and surgical intervention, from self-tonometry with the Icare ${ }^{\circledR}$ HOME tonometer. Paired t-tests compare the ranges between self-tonometry performed during the clinic office hours phasing and all day self-tonometry measurements within individuals.

\begin{tabular}{lllll}
\hline Diagnosis & Surgery & $\begin{array}{l}\text { Self-tonometry } \\
\text { office range IOP } \\
(\mathrm{mmHg})\end{array}$ & $\begin{array}{l}\text { Self-tonometry } \\
\text { single day range } \\
\text { IOP }(\mathrm{mmHg})\end{array}$ & $\begin{array}{l}\text { Peak range } \\
\text { IOP }(\mathrm{mmHg})\end{array}$ \\
\hline OHT $(\mathrm{N}=3)$ & & 4.4 & $9.2(\mathrm{p}=0.02)$ & $12.2(\mathrm{p}=0.00 \mathrm{I})$ \\
ACG $(\mathrm{N}=\mathrm{I})$ & No trabeculectomy $(\mathrm{N}=7)$ & 4.5 & 6.2 & 9.5 \\
OAG $(\mathrm{N}=14)$ & Trabeculectomy $(\mathrm{N}=7)$ & 4.7 & $7.3(\mathrm{p}<0.00 \mathrm{I})$ & $10.5(\mathrm{p}<0.00 \mathrm{I})$ \\
& All OAG $(\mathrm{N}=14)$ & 5.2 & $4.5(\mathrm{p}=0.36)$ & $6.1(\mathrm{p}=0.09)$ \\
& & $6.6(\mathrm{p}<0.00 \mathrm{I})$ & $9.3(\mathrm{p}<0.00 \mathrm{I})$ \\
\hline
\end{tabular}

IOP: intraocular pressure; OHT: ocular hypertension; ACG: angle closure glaucoma; OAG: open angle glaucoma.

conflicting results published in relation to the device's agreement with GAT. In relation to the Icare $^{\circledR}$ ONE tonometer, previous studies have reported variable agreement between self-tonometry and GAT. ${ }^{21-23}$ The agreement is generally good $(+/-1.2 \mathrm{~mm} \mathrm{Hg}<20 \mathrm{~mm} \mathrm{Hg}$ and $+/-2.2 \mathrm{~mm} \mathrm{Hg}$ $\geq 20 \mathrm{~mm} \mathrm{Hg}$ ) within the advised established corneal thicknesses between 500 and $600 u$ um. $^{24-28}$ The first part of this study is a simple observer comparison study between selftonometry with the updated Icare $^{\circledR}$ model, Icare $^{\circledR}$ HOME, and 'gold standard' GAT.

We observed good agreement between the Icare $^{\circledR}$ HOME and GAT at low IOPs, but with a progressive over-reading for the Icare ${ }^{\circledR}$ HOME for IOPs greater than $15 \mathrm{~mm} \mathrm{Hg}$. This could be partly explained by the inclusion of two patients with a CCT of $>600 \mathrm{um}$, and therefore outside the recommended range of 500-600um for the device. This is also in agreement with previous reports, which found Icare ${ }^{\circledR}$ devices to produce an overestimation with higher IOPs, largely due to thick corneas ${ }^{15,19,24,25,29,30}$

In order for 'home' IOP monitoring to become commonplace, the process must provide additional benefits over those provided by clinic GAT phasing. One such benefit is the potential for extra readings out with clinic hours. This could provide additional relevant information that may influence a patient's diagnosis or management. On the days when the patients performed home phasing without supervision (i.e. days two and three) only half of the readings were taken within standard office hours (i.e. 09:00-17:00). These readings out with standard office hours revealed an increase in the peak IOP recorded, (single day clinical hour phasing peak was $16.7 \mathrm{~mm} \mathrm{Hg}$, vs $18.5 \mathrm{~mm} \mathrm{Hg}$ over three days home phasing $(p=0.24))$. This is in agreement with Querat and $\mathrm{Chen}^{31}$ who noted $16 \%$ of 46 subjects undertaking self-tonometry had IOP peaks outside clinic hours. In addition the range of IOPs recorded were almost twice as large with home monitoring (range of $9.8 \mathrm{~mm} \mathrm{Hg}$ with home phasing vs $5.0 \mathrm{~mm} \mathrm{Hg}$ with clinic hours). There are several studies that have shown a greater fluctuation in the range of IOPs when measured out of office hours. ${ }^{32,33}$ Arora et al. ${ }^{34} 2015$ identified that, for glaucoma patients, the mean diurnal IOP and IOP fluctuations with GAT (07:00-22:00) were greater compared to standard office hours (09:00-17:00), and peak IOP measurements occurred outside office hours in two-thirds of patients. Our study identified a higher range of IOPs compared to Arora et al 2015, when comparing home phasing (00:00-24:00) and clinic hours phasing (09:00-16:30). This is possibly due to the greater time range available to the patients completing home phasing within our study. A study using the Icare $^{\circledR}$ ONE also identified higher IOP spikes outside office hours, with $50 \%$ of patient peak IOP values occurring outside office hours. ${ }^{35}$

Although in this study no evaluation was possible in regards to the accuracy of self-tonometry when out of the clinic and away from clinician supervision, it is worth noting that all 18 patients were classed as competent within office hours and their self-administered tonometry measurements were satisfactorily comparable to the optometrists Icare $^{\circledR}$ HOME measurement and GAT.

The larger IOP range from our findings concurs with the conclusion that home tonometry is a valid method for detecting diurnal fluctuation outside normal clinic hours. In a recently published study by Huang et al. ${ }^{36}$ 27 patients completed self-tonometry over 40 days. Using four readings per day, they reported 'correlation' of the IOP variation comparing the first day, first two days, first three days and so on with the results over 40 days. Not-surprisingly they reported increasing agreement as the number of measurement days increased with the range of IOP recorded over 40 days. Moderately good agreement was found between three days of measurements compared with 40 with a further small increase to seven days after which any increase was minimal. ${ }^{36}$ 
An additional key finding from this study was the consistency of IOP range in those eyes that had prior trabeculectomy surgery. The range did not increase with greater observation timing. This suggests the home tonometry may be generating accurate readings and also confirms prior reports of the effect of trabeculectomy surgery on IOP range. ${ }^{37}$

\section{Cost effectiveness}

The cost effectiveness of implementing a system in which home phasing with self-tonometry replaces inclinic phasing needs to be assessed. From a patient perspective, the time taken to learn self-tonometry versus the time taken to do all day phasing in a clinic seems a clear benefit. For the working population in particular, the time required for clinic phasing can be extremely inconvenient, whereas self-phasing may enable patients to continue their daily activities with minimal disruption. However, from the clinician perspective the time considerations may not be dissimilar.

The proportion of patients who successfully complete phase one should also be considered. Our previous study found that $74 \%$ of subjects were able to correctly perform self-tonometry. ${ }^{15}$ This agrees with other similar reports with the Icare $^{\circledR}$ HOME. ${ }^{18}$

Additional considerations include the overheads of clinical space (for example, patients need to have a place to wait during all day phasing), equipment maintenance and provision (slit lamp and GAT vs Icare ${ }^{\circledR}$ HOME), consumable use (anaesthetic drops and GAT tonometer heads vs probes and batteries for the Icare $^{\circledR}$ HOME). We believe many of these will not significantly vary between the two methods, therefore suggesting that the use of self-tonometry does not currently appear to be advantageous in terms of overall cost differences.

In conclusion, we previously demonstrated that $74 \%$ of patients can use the Icare ${ }^{\circledR}$ HOME tonometer successfully. ${ }^{15}$ With this latest study we have built on those findings, demonstrating that self-tonometry within a 'home' environment is possible and a viable alternative to in-office clinic GAT IOP phasing. Furthermore, the findings from this study suggest self-tonometry home phasing is beneficial in demonstrating higher peak and trough IOPs, thereby providing more detailed ocular health information. The cost-effectiveness of this approach has yet to be fully addressed, but initial review indicates that cost comparisons are similar.

\section{Acknowledgement}

We are indebted to the International Glaucoma Association who kindly provided the grant enabling this research.

\section{Author contributions}

This study was conceived and designed by Ian Murdoch and John Lawrenson. Emily McGarva, Jane Farr, Priya Dabasia, John Lawrenson and Ian Murdoch participated in analysis, interpretation and manuscript preparation.

\section{Declaration of conflicting interests}

The author(s) declared no potential conflicts of interest with respect to the research, authorship, and/or publication of this article.

\section{Funding}

The author(s) disclosed receipt of the following financial support for the research, authorship, and/or publication of this article: This project was funded by a grant from the International Glaucoma Association.

\section{ORCID iD}

Ian E Murdoch (D) https://orcid.org/0000-0002-5453-0635

\section{References}

1. Coleman AL and Miglior S. Risk factors for glaucoma onset and progression. Surv Ophthalmol 2008; 53(Suppl. 1): S3-S10.

2. Liu JH, Zhang X, Kripke DF, et al. Twenty-four-hour intraocular pressure pattern associated with early glaucomatous changes. Invest Ophthalmol Vis Sci 2003; 44(4): 1586-1590.

3. The Advanced Glaucoma Intervention Study (AGIS): 7. The relationship between control of intraocular pressure and visual field deterioration - the AGIS investigators. Am J Ophthalmol 2000; 130(4): 429-440.

4. Gordon MO, Beiser JA, Brandt JD, et al. The Ocular Hypertension Treatment Study: baseline factors that predict the onset of primary open-angle glaucoma. Arch Ophthalmol 2002; 120(6): 714-720; discussion 829-830

5. Bengtsson B, Leske MC, Hyman L, et al. Fluctuation of intraocular pressure and glaucoma progression in the early manifest glaucoma trial. Ophthalmology 2007; 114(2): 205-209.

6. Musch DC, Gillespie BW, Niziol LM, et al. Intraocular pressure control and long-term visual field loss in the Collaborative Initial Glaucoma Treatment Study. Ophthalmology 2011; 118(9): 1766-1773.

7. Abraham LM, Epasinghe NC, Selva D, et al. Comparison of the ICare rebound tonometer with the Goldmann applanation tonometer by experienced and inexperienced tonometrists. Eye 2008; 22(4): 503-506.

8. Liu JH, Kripke DF, Twa MD, et al. Twenty-four-hour pattern of intraocular pressure in the aging population. Invest Ophthalmol Vis Sci 1999; 40(12): 2912-2917.

9. Wilensky JT, Gieser DK, Dietsche ML, et al. Individual variability in the diurnal intraocular pressure curve. Ophthalmology 1993; 100(6): 940-944.

10. Jensen AD and Maumenee AE. Home tonometry. Am J Ophthalmol 1973; 76(6): 929-932. 
11. Collins CC. Miniature passive pressure transensor for implanting in the eye. IEEE Trans Biomed Eng 1967; 14(2): 74-83.

12. Bliziotis IA, Destounis A and Stergiou GS. Home versus ambulatory and office blood pressure in predicting target organ damage in hypertension: a systematic review and meta-analysis. J Hypertens 2012; 30(7): 1289-1299.

13. Zhu $\mathrm{H}$, Zhu $\mathrm{Y}$ and Leung SW. Is self-monitoring of blood glucose effective in improving glycaemic control in type 2 diabetes without insulin treatment: a metaanalysis of randomised controlled trials. BMJ Open 2016; 6(9): e010524.

14. Kontiola AI. A new induction-based impact method for measuring intraocular pressure. Acta Ophthalmol Scand 2000; 78(2): 142-145.

15. Dabasia PL, Lawrenson JG and Murdoch IE. Evaluation of a new rebound tonometer for self-measurement of intraocular pressure. $B r J$ Ophthalmol 2016; 100(8): 1139-1143.

16. Dielemans I, Vingerling JR, Hofman A, et al. Reliability of intraocular pressure measurement with the Goldmann applanation tonometer in epidemiological studies. Graefes Arch Clin Exp Ophthalmol 1994; 232(3): 141-144.

17. Bland JM and Altman DG. Statistical methods for assessing agreement between two methods of clinical measurement. Lancet 1986; 1(8476): 307-310.

18. Pronin S, Brown L, Megaw R, et al. Measurement of intraocular pressure by patients with glaucoma. JAMA Ophthalmol 2017; 135(10): 1-7.

19. Takagi D, Sawada A and Yamamoto T. Evaluation of a new rebound self-tonometer, Icare HOME: comparison with Goldmann applanation tonometer. J Glaucoma 2017; 26(7): 613-618.

20. Mudie LI, LaBarre S, Varadaraj V, et al. The Icare HOME (TA022) Study: performance of an intraocular pressure measuring device for self-tonometry by glaucoma patients. Ophthalmology 2016; 123(8): 1675-1684.

21. Tan S, Yu M, Baig N, et al. Agreement of patientmeasured intraocular pressure using rebound tonometry with Goldmann applanation tonometry (GAT) in glaucoma patients. Sci Rep 2017; 7: 42067.

22. Asrani S, Chatterjee A, Wallace DK, et al. Evaluation of the ICare rebound tonometer as a home intraocular pressure monitoring device. J Glaucoma 2011; 20(2): 74-79.

23. Moreno-Montanes J, Martinez-de-la-Casa JM, Sabater AL, et al. Clinical evaluation of the new rebound tonometers Icare PRO and Icare ONE compared with the Goldmann tonometer. J Glaucoma 2015; 24(7): 527-532.

24. Halkiadakis I, Stratos A, Stergiopoulos G, et al. Evaluation of the Icare-ONE rebound tonometer as a self-measuring intraocular pressure device in normal subjects. Graefes Arch Clin Exp Ophthalmol 2012; 250(8): 1207-1211.
25. Rosentreter A, Jablonski KS, Mellein AC, et al. A new rebound tonometer for home monitoring of intraocular pressure. Graefes Arch Clin Exp Ophthalmol 2011; 249(11): 1713-1719.

26. Sakamoto M, Kanamori A, Fujihara $M$, et al. Assessment of IcareONE rebound tonometer for selfmeasuring intraocular pressure. Acta Ophthalmol 2014; 92(3): 243-248.

27. Munkwitz S, Elkarmouty A, Hoffmann EM, et al. Comparison of the iCare rebound tonometer and the Goldmann applanation tonometer over a wide IOP range. Graefes Arch Clin Exp Ophthalmol 2008; 246(6): 875-879.

28. Tamcelik N, Atalay E, Cicik E, et al. Comparability of Icare PRO rebound tonometer with Goldmann applanation and noncontact tonometer in a wide range of intraocular pressure and central corneal thickness. Ophthalmic Res 2015; 54(1): 18-25.

29. Chen E, Querat L and Akerstedt C. Self-tonometry as a complement in the investigation of glaucoma patients. Acta Ophthalmol 2016; 94(8): 788-792.

30. Termuhlen J, Mihailovic N, Alnawaiseh M, et al. Accuracy of measurements with the iCare HOME rebound tonometer. J Glaucoma 2016; 25(6): 533-538.

31. Querat L and Chen E. Monitoring daily intraocular pressure fluctuations with self-tonometry in healthy subjects. Acta Ophthalmol 2017; 95(5): 525-529.

32. Moodie J, Wilde C, Rotchford AP, et al. 24-Hour versus daytime intraocular pressure phasing in the management of patients with treated glaucoma. Br J Ophthalmol 2010; 94(8): 999-1002.

33. Tajunisah I, Reddy SC and Fathilah J. Diurnal variation of intraocular pressure in suspected glaucoma patients and their outcome. Graefes Arch Clin Exp Ophthalmol 2007; 245(12): 1851-1857.

34. Arora T, Bali SJ, Arora V, et al. Diurnal versus officehour intraocular pressure fluctuation in primary adult onset glaucoma. J Optom 2015; 8(4): 239-243.

35. Sood V and Ramanathan US. Self-monitoring of intraocular pressure outside of normal office hours using rebound tonometry: initial clinical experience in patients with normal tension glaucoma. J Glaucoma 2016; 25(10): 807-811.

36. Huang J, Katalinic P, Kalloniatis M, et al. Diurnal intraocular pressure fluctuations with self-tonometry in glaucoma patients and suspects: a clinical trial. Optom Vis Sci 2018; 95(2): 88-95.

37. Medeiros FA, Pinheiro A, Moura FC, et al. Intraocular pressure fluctuations in medical versus surgically treated glaucomatous patients. J Ocul Pharmacol Ther 2002; 18(6): 489-498. 